\title{
A Multiplicative Human Steering Control Model
}

\author{
Miguel Martínez-García, Timothy Gordon \\ School of Engineering \\ University of Lincoln \\ Brayford Pool, LN6 7TS, UK \\ Email: \{mmartinez, tgordon\}@lincoln.ac.uk
}

\begin{abstract}
A non-linear, yet simple, multiplicative humancontrol model is developed by studying the statistical properties of human subjects' motor response to a visual input; statistical analysis of the magnitude of the steering angle, from subjects performing a tracking task with a steering wheel, shows that the data is consistent with a log-normal distribution. Thus the possibility of modelling human-control as a multiplicative process, that replicates the statistical properties found in the humanoperator is considered. The proposed multiplicative controller is contrasted with real data and with the Crossover Model. This research has potential applications in a wide range of fields, from human performance modelling to the development of humanmachine interfaces, particularly in the application of ground vehicle automation.
\end{abstract}

\section{INTRODUCTION}

Nearly every existing human-control model incorporates a series of parameters [1], which not always have physiological interpretation. Usually, with the intention of reproducing the control actions of the human-operator as close as possible, the parameters are adjusted to match the responses of human subjects. This approach, while not incorrect, has one inconvenience: often it is difficult to tell to which extent an accurate model representation is the result of a good model, or of the optimization procedure to fit its parameters [2]. This is especially true for models with many parameters. The most pre-eminent example is the use of neural networks to model the human [3]; neural networks can be used to model virtually any dynamical system. Although in many respects these models are very valuable, they do not produce insights on the characteristics of human-control. Furthermore, different subjects may use diverse control strategies that not necessary match the same model. At the same time, for sufficiently complex systems, alternative interpretations can explain the same observed effects.

In this paper, an statistical approach to biofidelic modelling, i.e. modelling human-operator behaviour in a faithful way biologically speaking, is introduced. The approach consists of considering a model to be biofidelic, when it reproduces some of the statistical properties of the human-operator in a particular task. The used methodology is consistent with the Principle of Maximum Entropy [4], which states that given some initial data, the distribution that better describes the data under some specified constraints, is the one with maximum entropy. In this paper, the intended task is to organize the observed results into a model that is as simple as possible.
A factor of motivational value for this research is that, recent and relevant studies in neuroscience [5], have shown that several aspects regarding the dynamics of the brain are characterized by long-tailed probability distributions such as the log-normal. One example is the distribution of firing rates in cortical neurons. In this paper, the possibility of steering wheel control in a tracking task to be characterized by a log-normal distribution is considered. Given that the log-normal distribution arises as a result of multiplicative processes, a multiplicative humancontrol model is proposed. The model is compared with data from human subjects and with a standard approach in humanperformance modelling: the Crossover Model [6].

For this research, data collected from human subjects in compensatory and pursuit tracking tasks, and using a steering wheel as control device, is utilized [7].

An accurate representation of the human-operator is an important part in the design of human-computer interfaces. This research is aimed towards biofidelic formulation of humancontrol models, in particular to their application to ground vehicle automation and driver assistance technologies.

The discussion is organized as follows: In Section II the needed theoretical background is presented. Section III introduces the human tracking experiments from which the data was collected. By analyzing the data, a novel approach to model the human-operator is proposed in Section IV. Lastly, in Section V, conclusions are drawn and potential extensions of this work are suggested.

\section{TheORETICAL BACKGROUND}

\section{A. Log-normal distributions and multiplicative dynamics}

Consider a multiplicative process of the form

$$
G_{t_{k}}=\xi_{t_{k}} G_{t_{k-1}}=G_{t_{0}} \prod_{i=t_{1}}^{t_{k}} \xi_{i},
$$

where $\left(t_{i}, t_{i+1}\right) \subset \mathbb{R}^{+} \cup\{0\}$ are equispaced time intervals and $G_{t_{0}}>0, \xi_{i}>0$. Taking natural logarithms in (1) results in

$$
\log G_{t_{k}}=\log G_{t_{0}}+\sum_{i=t_{1}}^{t_{k}} \log \xi_{i} .
$$

If $\xi_{i}$ are identically distributed and independent random variables, the Central Limit Theorem (CLT) applies to the summation term of (2) and, for large enough $k, \log G_{t_{k}}$ will be approximately normally distributed. Therefore, $G_{t_{k}}$ will follow approximately a log-normal distribution. In general, the 
discrete observations of a time series $\left(\xi_{i}\right)$ are not independent, unless the series is a Markov process. However, an alternate formulation of the CLT that applies for the analysis of arbitrary time series can be employed [8].

Definition. $m$-dependent random variables:

A sequence of random variables $X_{i}$ for $i=1 \ldots N$ is said to be $m$-dependent when $|i-j|>m$ implies that $X_{i}$ and $X_{j}$ are independent. If the sequence $\left\{X_{i}\right\}_{i=1 \ldots N}$ is understood as a time series, $m$-dependence means that events occurring at least $m$ time steps away are independent.

Theorem. Central Limit Theorem for $m$-dependent random variables:

If $X_{i}$ is a sequence of $m$-dependent random variables with expected value $E\left[X_{i}\right]=0$ and $E\left[\left|X_{i}\right|^{3}\right]<\infty$, then

$$
Y_{\infty}=\lim _{N \rightarrow \infty} N^{-1 / 2} \sum_{i=1}^{N} X_{i}
$$

is normally distributed with $E\left[Y_{\infty}\right]=0^{1}$. In particular, for large $N, Y_{N}=\sum_{i=1}^{N} X_{i}$ approximates a normal distribution of zero expected value.

This result is more a technical requirement than a limitation. In practice, a given time series - predicted to arise from a multiplicative process - is fitted to a log-normal probability distribution after under-sampling the series with different values of $m$. If the results are equivalent for $m \geq \hat{m}$, the original time series can be considered $\tilde{m}$-dependent; the under-sampled time series can then be treated as a Markov process.

The potential interest in the log-normal distribution, with the purpose of modelling the human-operator, relies on two points. One is that the log-normal is a distribution of maximum entropy (Section II-B). The second point is that, several research studies in neuroscience have brought attention to the fact that, multiple aspects of the Central Nervous System (CNS) dynamics are log-normally distributed. For instance, log-normal distributions yield the best fit for the distribution of neuron firing rates in the cortex [9]. Another example is from research in the activity of neurons in the lower spine of turtles while engaging in rhythmic scratching; this appears to be also distributed according to a log-normal [10]. And interestingly, in [11] it is shown that, visual perceptual learning can be described as a process in which the human observer reduces the magnitude of internal multiplicative noise. Additionally, in [5], it is suggested that complex interconnected biological systems produce multiplicative and not additive interactions.

\section{B. Maximum entropy probability distribution}

The Principle of Maximum Entropy [4] asserts that, when determining which probability distribution explains better some data, and under some established constraints, the probability distribution with the maximum uncertainty should be chosen. For continuous probability distributions, the differential entropy is considered as measure of uncertainty. In the case of a positive

\footnotetext{
${ }^{1}$ There is also an expression for the variance of $Y_{\infty}$ in [8].
}

random variable $X$ with probability density function (PDF) $f_{X}$, the differential entropy is:

$$
H(X)=-\int_{0^{+}}^{\infty} f_{X}(x) \log \left(f_{X}(x)\right) d x .
$$

Assuming the constraints: $X>0, E[\log (X)]=\mu$ and $E\left[(\log (X)-\mu)^{2}\right]=\sigma^{2}$, the PDF obtained by maximizing expression (4) is the log-normal:

$$
f_{X}(x)=\frac{1}{x \sqrt{2 \pi \sigma^{2}}} e^{-\frac{(\log (x)-\mu)^{2}}{2 \sigma^{2}}} .
$$

The approach is equivalent to introducing the least possible number of constraints. In this research, the human-operator is modelled based on partial information; only data from recordings of operator's responses in a control task are used. It is considered that almost no knowledge about the features of human-control are known; in reality this turns out to the case. Thus the methodology is consistent with the Principle of Maximum Entropy.

\section{Bootstrap test for goodness-of-fit}

Once a probability distribution is selected to be representative of a data set $\mathbb{X}$, and its parameters are fitted to the data giving a PDF $f_{\mathbb{X}}$ and a cumulative distribution function (CDF) $F_{\mathbb{X}}$, in order to quantify the goodness-of-fit the following bootstrap method can be used [12]:

(i) Determine the distance between the fitted probability distribution and the empirical data by using some statistical measure, for example the Kolmogorov-Smirnov distance can be used:

$$
D=\max _{x \in \mathbb{X}}\left|F_{\mathbb{X}}(x)-\tilde{F}_{\mathbb{X}}(x)\right|
$$

where $\tilde{F}_{\mathbb{X}}$ is the empirical CDF.

(ii) Generate a set of pseudo-random numbers $\mathbb{Y}$ distributed according to $F_{\mathbb{X}}$.

(iii) The parameters of the chosen probability distribution are fitted again to the new artificial data $\mathbb{Y}$, obtaining a new $\mathrm{CDF} F_{\mathbb{Y}}$.

(iv) The Kolmogorov-Smirnov distance is calculated for the artificial data set $\mathbb{Y}$,

$$
d=\max _{y \in \mathbb{Y}}\left|F_{\mathbb{Y}}(y)-\tilde{F}_{\mathbb{Y}}(y)\right| .
$$

(v) Steps (ii) to (iv) are iteratively repeated many times (say $10000-100000$ times) generating a set of distances $\mathbb{D}=$ $\left\{d_{1}, d_{2}, \ldots, d_{N}\right\}$

(vi) The $p$-value of the goodness-of-fit test is estimated according to

$$
p=\frac{\#\{d \in \mathbb{D} \mid d>D\}}{N}
$$

where \# denotes the cardinality of the specified set, that is, $p$ is the proportion of distances in $\mathbb{D}$ greater than $D$. If $p>0.1$ the goodness-of-fit test rejects the null hypothesis of the data not belonging to the specified distribution; the data can be explained by the distribution considered. 


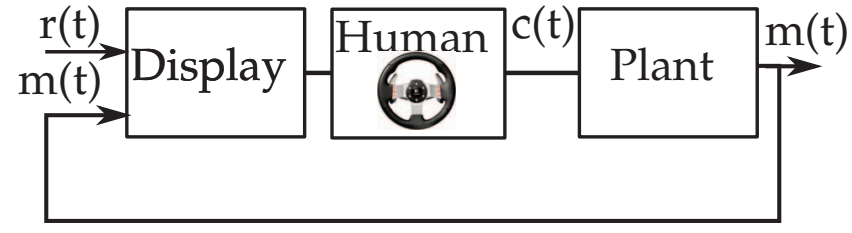

Fig. 1: Man-machine control loop in the experimental setup used to collect the data. In compensatory mode, the display presents a fixed reference circle at the center of the display, and a moving solid dot with position $e(t)$. In pursuit mode, the reference circle moves with position $r(t)$. The solid dot has position $m(t)$ thus the error is perceived as the difference between $r(t)$ and $m(t)$.

\section{Human Tracking Task Data Collection}

Ten subjects, of ages between 22 and 33 and mixed genders, participated in a series of data collection experiments performing a tracking task. The goal of the subjects was to control a one-dimensional plant with a steering wheel. The data was collected in compensatory and in pursuit mode [7]. In compensatory mode, only one object - a solid dot - moved in the screen with respect to a fixed reference point - a circle at the center of the display - along the horizontal direction. The distance from the dot to the circle represented the error $e(t)$. The human subjects were requested to keep the error as small as possible by using the control device. The error was induced by a forcing function $r(t)$. The moving dot, responded to the human manipulative control actions $c(t)$ according to a transfer function $Y_{p}$, which determined the plant dynamics $m(t)$ (Fig. 1). In compensatory mode the plant dynamics are not visualized by the human-operator, which only has access to the relative error $e(t)=r(t)-m(t)$.

In pursuit mode, the reference circle is not fixed in the display but moved according to the forcing function. The position or lateral offset on the display of the other moving element is controlled by the plant dynamics $m(t)$ (Fig. 1). Although neither of the moving elements directly represents the error in pursuit mode, the human perceives the error as the relative difference in position between the moving reference point, with position determined by the forcing function, and the solid dot, controlled by the plant dynamics. The task of the human was to reduce their distance.

For each subject two tracking events were recorded of $90 \mathrm{~s}$ duration. Half of the subjects performed the tracking task in compensatory mode and the other half in pursuit mode. In both modes, the plant dynamics were governed by the transfer function:

$$
Y_{p}(s)=\frac{K}{s(T s+1)},
$$

with $K=5, T=0.1$.

The forcing function $r(t)$ was composed of a sum of sinusoids with a range of frequencies $f_{k}=0.01-20 \mathrm{~Hz}$ :

$$
r(t)=\sum_{f_{k} \in\{0.01 \ldots 20\}} e^{-4 f_{k}} \sin \left(f_{k} \cdot 2 \pi t-\varphi_{k}\right),
$$

where $\varphi_{k} \in[-\pi, \pi]$ is a randomized phase for each summation term. With this choice, the amplitude is negligible for frequencies outside of the range where a human-operator can perform adequate control. Considering quasi-random forcing functions, humans can't perform tracking with acceptable performance for frequencies greater than approximately $1 \mathrm{~Hz}$ [13].

Regarding the steering wheel, it was calibrated to allow a total of 900 degrees of rotation lock to lock. For each of the events there was recorded: the forcing function, the plant output, the error signal and the human response, which was the normalized steering wheel angle from -1 ( 450 degrees to the left) to 1 (450 degrees to the right). For each of the $90 \mathrm{~s}$ events, the initial $20 \mathrm{~s}$ and the last $10 \mathrm{~s}$ were excluded. Thus from each event, only $60 \mathrm{~s}$ of data are analyzed. The recorded data were sampled at $100 \mathrm{~Hz}$.

These experiments were part of a more extensive data collection project, which included fractional order transfer functions among other variants [7].
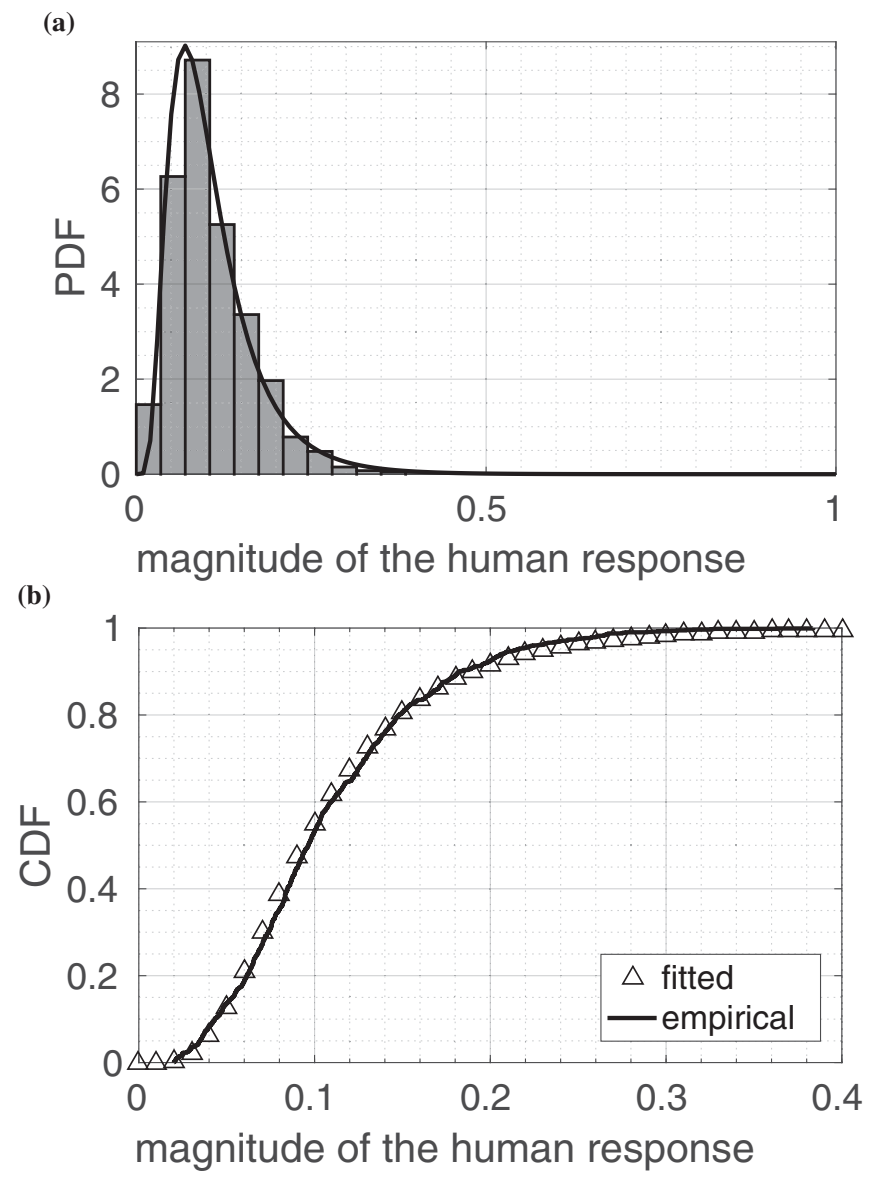

Fig. 2: (a) Normalized histogram for the responses of all the subjects combined - recorded from the steering wheel sensor - along with the fitted log-normal PDF. (b) Fitted CDF and empirical values. The fitted parameters for the log-normal are $\mu=-2.368$, with $95 \%$ confidence interval $[-2.401,-2.337]$, and $\sigma=0.550$, with confidence interval $[0.528,0.573]$. 


\section{Model Proposal And VALIDATION}

\section{A. Distribution of the human-operator responses}

The uncertainty in the response of a human-operator to an input, is a challenge when trying to identify the operator as a deterministic actuator. The exact input to which the operator responds is itself generally unknown. Hence, the possibility of characterizing the responses of the human-operator in a probabilistic manner is examined.

By analyzing the magnitude of the human responses $(|c(t)|)$ to the displayed error, empirically one sees that it has the shape of a skewed distribution (Fig. 2a). To visualize the data, the steering responses of all the ten subjects were combined, after being under-sampled to a sampling rate of $1 \mathrm{~Hz}$. This was done in order to confirm that the series is $m$-dependent (Section II-A). For $m>100$ no appreciable differences were observed visually or numerically in the results. Additionally, values such that $|c(t)|<0.02$ were discarded. This threshold was set because no evident pattern was found in the data for smaller magnitudes, and because the log-normal is defined over a positive support.

The parameters of the log-normal distribution were fitted to the data through maximum likelihood estimation. Subsequently, a goodness-of-fit test was performed (Section II-C) from which a $p$ value of 0.317 is obtained. Between the individual subjects the fitted parameters present some variability (Table I), although the values are comparable; since different subjects employ different control strategies and have different skill levels, this was not considered surprising.

Thus the statistical analysis indicates that, the magnitude of the control responses over a threshold, can be explained as a process distributed according to a log-normal. This result motivates the possibility of modelling the human-operator responses as a multiplicative process.

\section{B. Multiplicative human-control model}

A non-linear multiplicative human-control (MHC) model is here proposed. In the MHC model, the control response $C\left(t_{k}\right)$, according to a time discretization $t_{1}, t_{2}, \ldots, t_{k}, \ldots, t_{f}$ with fixed time-step $s=t_{k}-t_{k-1}$, is defined as

$$
C\left(t_{k}\right) \equiv K_{H} S_{t_{k}} H_{t_{k}}
$$

where $K_{H}$ represents the neuromuscular gain, $H_{t_{k}}$ is the magnitude of the response intent of the human-operator, and $S_{t_{k}}$ is a sign function that determines in which direction the response is applied. $H_{t_{k}}$ is designed to simulate a multiplicative process dependent on the previous observations of the error signal, with multiplicative factor $\xi_{t_{k}}:\left(e_{0}, \ldots, e_{t_{k}}\right) \longrightarrow(0, \infty)$ :

$$
H_{t_{k}} \equiv \xi_{t_{k}} H_{t_{k-1}}
$$

With this scheme, different functions $S_{t_{k}}$ and $\xi_{t_{k}}$ can be specified. Considering that humans act after a certain neuromuscular lag $\tau$ [7], that can be represented as the number of discretization steps $\rho=\tau / s, S_{t_{k}}$ and $\xi_{t_{k}}$ are defined thus:

$$
S_{t_{k}} \equiv \operatorname{sgn}\left(L^{\rho}\left\{e_{t_{k}}\right\}\right)
$$

\begin{tabular}{l|cc|ccc}
\hline & \multicolumn{2}{c}{ human } & \multicolumn{3}{c}{ virtual human } \\
\hline Subject & $\mu$ & $\sigma$ & gain $K_{H}$ & $\mu$ & $\sigma$ \\
\hline S1 & -2.641 & 0.406 & 10.570 & -2.920 & 0.525 \\
S2 & -2.274 & 0.547 & 18.940 & -2.617 & 0.627 \\
S3 & -2.289 & 0.603 & 26.370 & -2.602 & 0.699 \\
S4 & -2.036 & 0.506 & 15.626 & -2.451 & 0.588 \\
S5 & -2.311 & 0.566 & 21.650 & -2.640 & 0.587 \\
S6 & -2.354 & 0.401 & 9.498 & -2.862 & 0.474 \\
S7 & -2.602 & 0.459 & 11.417 & -2.926 & 0.578 \\
S8 & -2.338 & 0.652 & 23.759 & -2.817 & 0.617 \\
S9 & -2.425 & 0.458 & 18.385 & -2.827 & 0.618 \\
S10 & -2.406 & 0.617 & 15.565 & -2.709 & 0.514 \\
\hline
\end{tabular}

TABLE I: Fitted parameters for the log-normal distribution (5) tabulated per subject. The table shows the results for the data collected from the human subjects, and for the artificial data obtained by running the multiplicative control model - with the same quasi-random forcing function $r(t)$ that was presented to each subject. For the case of the artificial data, the fitted gain of the multiplicative control model $K_{H}$ is also shown.

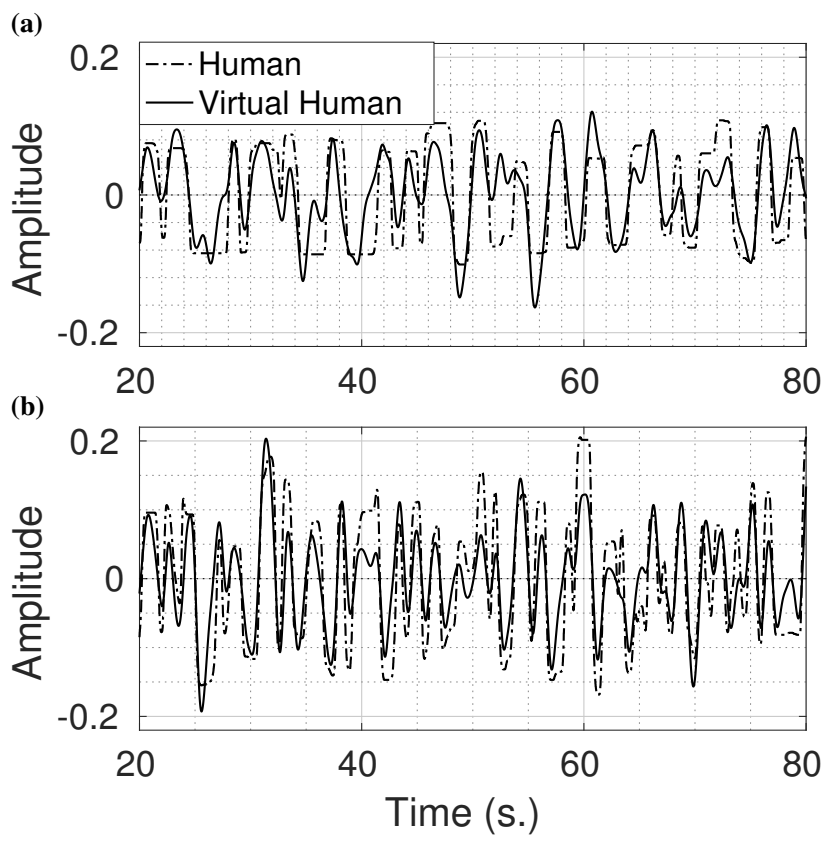

Fig. 3: Amplitude of the steering signal for two different randomized forcing functions $r(t)$ in (a) and (b). The two curves in each plot represent the response of a human subject $c(t)$ and the response $C(t)$ of the MHC model (14) with fitted $K_{H}$ to match the subjects' response: (a) $K_{H}=10.570$ and (b) $K_{H}=18.385$.

and

$$
\xi_{t_{k}} \equiv L^{\rho}\left\{\left|\frac{e_{t_{k}}}{e_{t_{k-1}}}\right|^{\nu}\right\}
$$

where $\nu>0$ (here $\nu=0.9$ ) and $L^{\rho}$ is the back-shift operator $\left(L^{\rho}\left\{e_{t_{k}}\right\}=e_{t_{k-\rho}}\right)$. The infrequent case where $S_{t_{k}}=0$ or $L^{\rho}\left\{e_{t_{k-1}}\right\}=0$ can be handled separately by assigning $\xi_{t_{k}}=$ 
$\xi_{t_{k-1}}$ or by setting an error threshold. For this particular choice of functions (12)-(13), the MHC model can be written as

$$
\begin{array}{r}
C\left(t_{k}\right) \equiv K_{H} \tilde{H}_{t_{k}} \\
\tilde{H}_{t_{k}} \equiv L^{\rho}\left\{\frac{e_{t_{k}}\left|e_{t_{k}}\right|^{\nu}}{\left|e_{t_{k-1}}\right|^{\nu+1}}\right\} \tilde{H}_{t_{k-1}}
\end{array}
$$

which is a non-linear three parameter model.

(a)

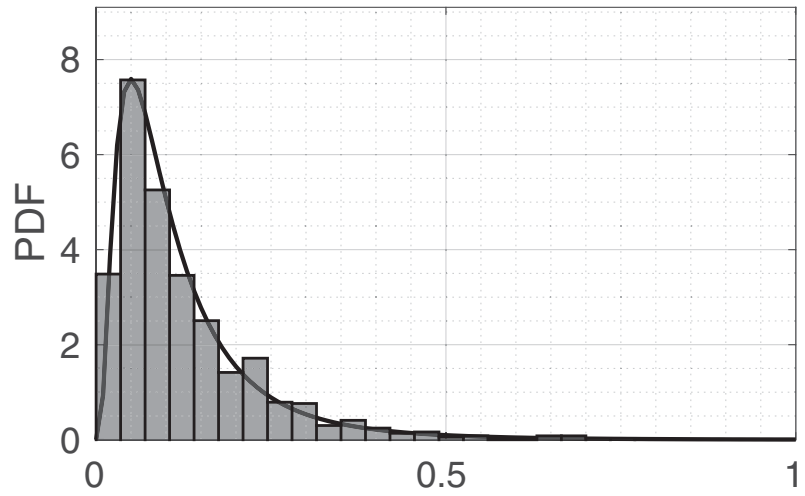

(b)
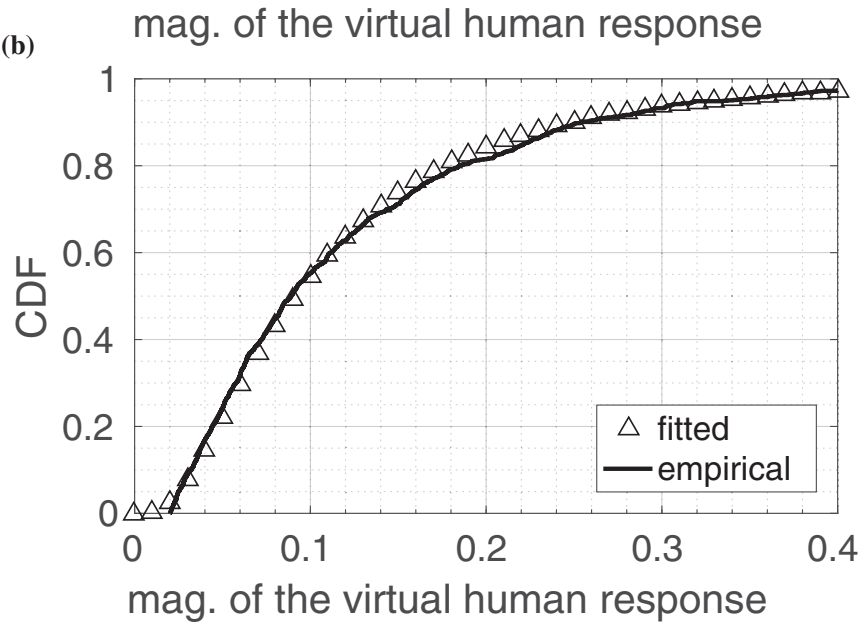

Fig. 4: (a) Normalized histogram of the virtual human (MHC) responses, for all the same instances of $r(t)$ that were presented to the ten subjects, and fitted PDF. (b) Fitted CDF and empirical values. The fitted parameters for the log-normal are $\mu=-2.395$, with $95 \%$ confidence interval $[-2.442,-2.347]$, and $\sigma=0.782$, with confidence interval $[0.751,0.816]$.

\section{Model Validation}

Since different subjects present distinct control strategies (Fig. 3), the gain $K_{H}$ was fitted to match the control responses of each subject through a Bayesian optimization algorithm (Table I). For example, the subject in Fig. 3a relies on lower amplitude and higher duration pulses, compared to the subject in Fig. 3b. The neuromuscular lag was also fitted from the subjects' data by a cross-correlation method [7] $(\tau \approx 0.361$ for the studied cases).

Once artificial data was generated by running the model, with the same randomized instances of Equation (9) (for $r(t)$ ) that were presented to each subject, the artificial data was fitted to a log-normal distribution. With this purpose, the same method used to fit the subjects' data was applied. For the combined data of all the simulations, the results are summarized in Fig. 4 A goodness-of-fit test results in a larger $p$-value compared to the human's data $(p=0.752)$. This was anticipated since the artificial data originates from a purely multiplicative process. The log-normal parameters for the particular instances of $r(t)$ of the virtual human were also calculated (Table I), showing values comparable to those from the subjects' responses.

The MHC model is here contrasted with a standard methodology in human-performance modelling, the Crossover Model [6]. The Crossover Model, describes the combined response of the human-operator and the plant to a input, with a human-machine system transfer function:

$$
Y_{h}(s) Y_{p}(s)=\frac{\omega_{c} e^{-\tau s}}{s},
$$

where $Y_{h}$ represents the human and $Y_{p}$ the plant. The model has two parameters: $\omega_{c}$ which is the combined human-plant open-loop gain, and $\tau$ the neuromuscular lag of the humanoperator. The Crossover Model has been widely used as a benchmark to validate other human-control approaches [14], [15]. For the presented application, the data generated from the virtual human is fitted very well by this model (Fig. 5). The Crossover Model only gives an adequate representation of the human-operator frequency response in the vicinity of the crossover frequency $\omega_{c}$ [7]. The MHC model presents Crossover Model behaviour over a wider frequency range. This may be caused by the lack of human internal noise in the MHC model and by limitations in the modelling approach.

\section{Critique}

Although the presented methodology offers satisfactory results, there are a number of discrepancies between the model output and the real data. Humans present a higher crossover frequency than that of the MHC model (Table II). This results in the MHC model having a higher root-mean-square error (RMSE) in the tracking task for the examined plant. Further, the given approach requires the use of a threshold in the data. Human-control is likely to be explained better below the threshold by another type of model. Moreover, the model assumes that the human manipulative actions are a stationary process, thus learning and adaptation are not considered.

\section{CONCLUSION}

This paper has presented a novel approach to humanperformance modelling. The approach develops models that present the same statistical properties found in the responses of the human-operator. In particular, for data obtained from human subjects in a tracking task, while controlling a plant with a steering wheel, it is shown that the data fits well a lognormal distribution. As the log-normal distribution arises from multiplicative processes, a non-linear multiplicative humancontrol (MHC) model is proposed. The model is compared to real data and to a prevailing model in the human-performance literature; it is shown that the MHC model is biofidelic and it fits well the predictions of the Crossover Model.

The results presented here correspond to a specific laboratory task with particular plant dynamics, forcing function and control 
(a)

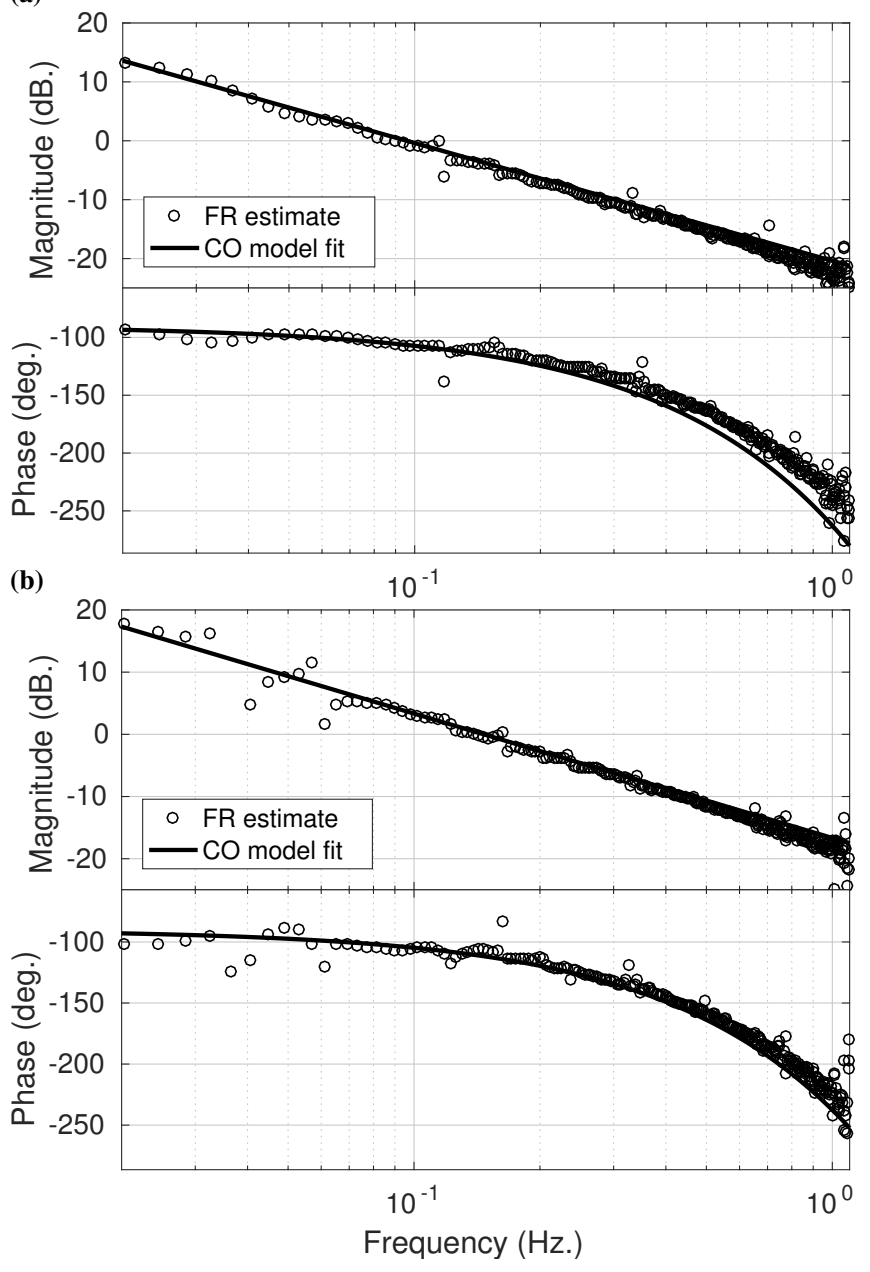

Fig. 5: Frequency response estimate of the virtual human (MHC) for the same events displayed in Fig. 3, (a) and (b) respectively. And frequency response of the Crossover Model with parameters $\omega_{c}$ and $\tau$ fitted to the data.

\begin{tabular}{l|cc|cc}
\hline & \multicolumn{2}{c}{ human } & \multicolumn{2}{c}{ virtual human } \\
\hline Subject & $\omega_{c}$ & RMSE & $\omega_{c}$ & RMSE \\
\hline S1 & 0.108 & $3.506 \times 10^{-3}$ & 0.088 & $5.271 \times 10^{-3}$ \\
S2 & 0.287 & $3.055 \times 10^{-3}$ & 0.156 & $4.914 \times 10^{-3}$ \\
S3 & 0.381 & $2.340 \times 10^{-3}$ & 0.207 & $3.311 \times 10^{-3}$ \\
S4 & 0.254 & $3.915 \times 10^{-3}$ & 0.128 & $6.510 \times 10^{-3}$ \\
S5 & 0.362 & $2.812 \times 10^{-3}$ & 0.174 & $4.233 \times 10^{-3}$ \\
S6 & 0.191 & $3.833 \times 10^{-3}$ & 0.080 & $6.111 \times 10^{-3}$ \\
S7 & 0.115 & $3.440 \times 10^{-3}$ & 0.095 & $5.170 \times 10^{-3}$ \\
S8 & 0.294 & $2.044 \times 10^{-3}$ & 0.189 & $3.119 \times 10^{-3}$ \\
S9 & 0.290 & $2.367 \times 10^{-3}$ & 0.150 & $3.583 \times 10^{-3}$ \\
S10 & 0.269 & $3.037 \times 10^{-3}$ & 0.128 & $4.691 \times 10^{-3}$ \\
\hline
\end{tabular}

TABLE II: Crossover frequency $\omega_{c}$ (in $\mathrm{Hz}$ ) and RMSE of the tracking task tabulated for each subject and for the virtual human. In the case of the virtual human, an identical function $r(t)(9)$ as in each of the events with human subjects was used. device and are not claimed to be universal. Although a steering wheel was used as control device, the data cannot be said to represent driver behaviour. Planned future work includes the study of how the results in this paper generalize to other type of plants and to driver behaviour.

Nevertheless, this research proposes that, knowing about the statistical organization of the human-operator responses, imposes constraints in human behaviour modelling and in the proposal of new theories to describe the principles of operation of human-control. Additionally, the statistical properties of the human-operator can be used to motivate new models and to further test the validity of the existing ones. As a minimum requirement, human-performance models should be asked to be statistically consistent with the responses of the humanoperator. It is noteworthy that such a straightforward approach can produce a model that on the one hand, reproduces the dynamical properties of the human-operator from a statistical point of view, and on the other hand is able to achieve stable control of the plant.

The aim of this research is to design human-machine interfaces that can predict the intentions of the human-operator, adapt to them and assist them in performing the underlying task.

\section{REFERENCES}

[1] R. J. Jagacinski and J. M. Flach, Control theory for humans: Quantitative approaches to modeling performance. CRC Press, 2003.

[2] M. Martínez-García, Y. Zhang, and T. Gordon, "Modeling lane keeping by a hybrid open-closed-loop pulse control scheme," IEEE Transactions on Industrial Informatics, vol. 12, no. 6, pp. 2256-2265, 2016.

[3] V. Mnih, K. Kavukcuoglu, D. Silver, A. A. Rusu, J. Veness, M. G Bellemare, A. Graves, M. Riedmiller, A. K. Fidjeland, G. Ostrovski et al., "Human-level control through deep reinforcement learning," Nature, vol. 518, no. 7540, pp. 529-533, 2015.

[4] E. T. Jaynes, "Information theory and statistical mechanics," Physical review, vol. 106, no. 4, p. 620, 1957

[5] G. Buzsáki and K. Mizuseki, "The log-dynamic brain: how skewed distributions affect network operations," Nature Reviews Neuroscience, vol. 15, no. 4, pp. 264-278, 2014.

[6] D. T. McRuer and H. R. Jex, "A review of quasi-linear pilot models," Human Factors in Electronics, IEEE Transactions on, no. 3, pp. 231-249, 1967.

[7] M. Martínez-García and T. Gordon, "Human control of systems with fractional order dynamics," in Systems, Man, and Cybernetics (SMC), 2016 IEEE International Conference on. IEEE, 2016, pp. 002866 002871 .

[8] W. Hoeffding, H. Robbins et al., "The central limit theorem for dependent random variables," Duke math. J, vol. 15, no. 3, pp. 773-780, 1948.

[9] A. Wohrer, M. D. Humphries, and C. K. Machens, "Population-wide distributions of neural activity during perceptual decision-making," Progress in neurobiology, vol. 103, pp. 156-193, 2013.

[10] P. C. Petersen and R. W. Berg, "Lognormal firing rate distribution reveals prominent fluctuation-driven regime in spinal motor networks," eLife, vol. 5, p. e18805, 2016

[11] J. Gold, P. Bennett, and A. Sekuler, "Signal but not noise changes with perceptual learning," Nature, vol. 402, no. 6758, pp. 176-178, 1999.

[12] W. Stute, W. G. Manteiga, and M. P. Quindimil, "Bootstrap based goodness-of-fit-tests," Metrika, vol. 40, no. 1, pp. 243-256, 1993.

[13] R. W. Pew, "Some history of human performance modeling," Integrated models of cognitive systems, pp. 29-44, 2007.

[14] C. C. Macadam, "Understanding and modeling the human driver," Vehicle System Dynamics, vol. 40, no. 1-3, pp. 101-134, 2003.

[15] T. Gordon and K. Srinivasan, "Modeling human lane keeping control in highway driving with validation by naturalistic data,' in Systems, Man and Cybernetics (SMC), 2014 IEEE International Conference on. IEEE, 2014, pp. 2507-2512. 